\title{
Interactions of interventional antiarrhythmic therapies with individual diseases and their substrate: the next challenge in improving patient outcomes
}

\author{
S. Saksena
}

Received: 6 January 2014 / Accepted: 7 January 2014 / Published online: 22 January 2014

(C) Springer Science+Business Media New York 2014

Interventions for the treatment of cardiac arrhythmias continued their global growth in robust fashion in 2013. Estimates for cardiac rhythm management devices continue to show projected growth rates from 9.3 to $11.1 \%$ annually depending on the global region [1]. Catheter ablation for the treatment of tachycardias could top 650,000 procedures annually by 2015 with an expected continuing annual growth rate of $13.4 \%$ [2, 3]. This growth is in part fueled by the expansion of the target patient populations and disease states for interventions. Accompanying the galloping growth of electrophysiological interventions now is the availability of increasingly robust datasets that can examine the nature and/or extent of the intervention with respect to patient outcomes. In the last few years, some important cautionary flags have begun to appear. High energy shocks, when frequently delivered by cardiac implantable electronic devices (CIEDs), can be associated with poorer survival in high-risk populations treated for the primary prevention of sudden death. Lower response rates to cardiac resynchronization therapy have been identified in patients with left ventricular leads implanted in many epicardial locations. Loss of atrial contractile function after extensive left atrial substrate ablation using radiofrequency energy has been documented.

It is of more than passing interest how we arrived at some of our current practices. While antitachycardia pacing reduced the need for low-energy shock therapy in slow and even fast ventricular tachycardia, technologic developments fostered empiric ATP programming. This expanded its usage to very early application virtually at tachycardia initiation, followed by a committed backup high-energy shock. Formal defibrillation threshold testing to choose shock energy is now being

S. Saksena $(\bowtie)$

Rutgers-Robert Wood Johnson Medical School,

161 Washington Valley Road, Suite 201, Warren, NJ, USA

e-mail: cmenj@aol.com increasingly avoided in clinical practice, also leading to the elimination of lower energy shocks. Such ubiquitous use has actually increased patient exposure to shocks for brief potentially self-terminating tachycardia events and supraventricular arrhythmias. Thus, the increasing absence of postoperative device testing, especially for optimizing tachycardia detection and tiered therapy algorithms, in favor of empiric high-energy shock delivery while reducing associated health care expenditures may have come at a greater price than previously imagined. Beyond mortality, inappropriate electrical therapy remains a surprisingly robust clinical challenge leading to many hospitalizations. It is now well recognized that fully $30-40 \%$ of cardiac resynchronization device implants performed solely by fluoroscopic imaging fail to elicit a meaningful response, even in carefully selected heart failure populations. Arrhythmia recurrence rates using anatomically driven atrial fibrillation ablation remain unacceptably high. In a disturbing report on real-world outcomes of 18,865 catheter ablation procedures in Germany, arrhythmia recurrence rates at 12 months ranged from $46 \%$ in atrial fibrillation to $17 \%$ in paroxysmal supraventricular tachycardia [4].

Despite these concerning outcomes, rays of hope have emerged, often from astute clinical observations. Simply delaying the response of electrical therapies in patients with implanted defibrillators has reduced patient shock exposure without compromising efficacy and safety in clinical trials. Left ventricular lead position has now emerged as an important determinant of response to CRT [5]. In fact, imagingguided placement may improve the degree and proportion of CRT response [6-8]. Mapping-guided ablation for ventricular tachycardia and paroxysmal supraventricular tachycardia has long been in vogue and may now impact atrial fibrillation ablation [9, 10].

The challenge between effectively eliminating and/or suppressing the arrhythmia and aggressiveness of the therapy is the daily dilemma faced by every interventional 
electrophysiologist. The core issues in these situations lie in the understanding of the pathogenesis, natural history, and outcomes of the untreated disease state before intervention and availability of robust data on the impact of the intervention on the structure and function of disease and its substrate. For example, we have now substantially expanded our electrophysiological understanding of the subpopulations of cardiac arrhythmias, whether congenital or acquired, associated with electrical, myocardial, or vascular disease or originating in specific cardiac locations. This proliferation of knowledge has not been matched by the concomitant delineation of the natural history or outcomes of individual arrhythmia states in modern times. We struggle with the overuse or underuse of CIEDs or ablation in many of these subpopulations. The expansion of map-guided ablation to substrate ablation occurred in the absence of thorough experimental investigation of the short- and long-term effects in relevant experimental models of disease state structure and function. Few electrophysiological investigations elucidate the non-arrhythmic functional status of the target tissue. Detailed hemodynamic studies have been scarce after interventions such as repeated shocks or ablation. Imaging studies are now becoming more prevalent and now identify serious issues for interventions such as silent cerebral emboli or impaired atrial contraction after ablation. This experience should suggest that such multidisciplinary study is necessary before new or expanded interventions are accepted into clinical practice. Far from stifling innovation, this would properly direct the development of interventions, maintain patient safety, and mitigate the increasing number of unsuccessful and extraordinarily expensive clinical trials. In a resource limited environment, this level of critical review appears necessary in 2014. It is therefore fitting that our first issue this year focuses on these needs using a large array of available tools and techniques to further our knowledge of interventional therapies.

As the Journal enters its 18th year, the excellent editorial support from our Springer editor, Ms. Lisa Noble and her team, remains a mainstay of our existence. The hard work and support of our reviewers, the guidance and input from our editorial board, and the tireless and unstinting efforts of all our editors makes our success possible. To all of them, I would take this opportunity to thank them for their enormous commitment to the Journal.

\section{References}

1. The Street.com, May 7, 2013.

2. Transparency Market Research, October 9, 2013

3. Personal communication, Boston Scientific Corporation.

4. J. Senges. Oral Presentation, 7th International Symposium on Cardiac Arrhythmias, Munich, September 20, 2013.

5. Mullens, W., Grimm, R. A., Vergat, T., Dresing, T., Starling, R. C., Wilkoff, B. L., et al. (2009). Insights from a cardiac resynchronization optimization clinic as part of a heart failure disease management program. Journal of the American College of Cardiology, 53, 765-773.

6. Khan, F. Z., Virdee, M. S., Palmer, C. R., Pugh, P. J., O’Halloran, D., Maros, E., et al. (2012). Targeted left ventricular lead placement to guide cardiac resynchronization therapy: the target study, a randomized, controlled trial. Journal of the American College of Cardiology, $59,1509-1518$

7. Saksena, S., Simon, A., Mathew, P., \& Nagarakanti, R. (2009). Intracardiac echocardiography guided cardiac resynchronization therapy: technique and clinical application. PACE, 32(8), 1030-1039.

8. Bai, R., Di Biase, L., Mohanty, P., Hesselson, A. B., De Ruvo, E., Gallagher, P. L., et al. (2011). Positioning of left ventricular pacing lead guided by intracardiac echocardiography with vector velocity imaging during cardiac resynchronization therapy procedure. Journal of Cardiovascular Electrophysiology, 22, 1034-1041.

9. Saksena, S., Skadsberg, N., Rao, H., \& Filipecki, A. (2005). Biatrial and 3-dimensional mapping of spontaneous atrial arrhythmias in patients with refractory atrial fibrillation. Journal of Cardiovascular Electrophysiology, 16(5), 494-504.

10. Narayan, S. M., Krummen, D. E., Clopton, P., Shivkumar, K., \& Miller, J. M. (2013). Direct or coincidental elimination of stable rotors or focal sources may explain successful atrial fibrillation ablation: on-treatment analysis of the CONFIRM trial (conventional ablation for AF with or without focal impulse and rotor modulation). Journal of the American College of Cardiology, 62(2), 138-147. 